\title{
Radio Spectral Variability of the Nearby GPS Galaxy PKS 1718-649
}

\author{
S. J. Tingay ${ }^{1}$ and M. de $\mathrm{Kool}^{2}$ \\ ${ }^{1}$ Centre for Austrophysics and Supercomputing, Swinburne University of Technology, \\ P.O. Box 218, Hawthorn, VIC 3122, Australia \\ stingay@astro.swin.edu.au \\ ${ }^{2}$ Research School of Astronomy and Astrophysics, Australian National University, \\ Cotter Road, Weston, ACT 2611, Australia \\ Received 2002 August 27, accepted 2003 January 6
}

\begin{abstract}
Data from new Australia Telescope Compact Array observations of a nearby gigahertz peaked spectrum radio source, PKS 1718-649, show significant variability both below and above the spectral turnover frequency during a nine month period. Simple models of synchrotron self-absorption (SSA) and free-free absorption (FFA) cannot explain the individual spectra or the spectral variability. Novel SSA or FFA models will need to be investigated in order to explain the complex variability in this source. Some potential explanations for the spectral variability are explored here but we can make no strong conclusion about which of SSA or FFA (or alternative mechanisms) causes the peaked spectrum in PKS 1718-649.
\end{abstract}

Keywords: galaxies: active — galaxies: individual (PKS 1718-649) — radio continuum: galaxies techniques: interferometric

\section{Introduction}

PKS 1718-649 is a low redshift example of a sub-class of active galactic nuclei (AGN), the gigahertz peaked spectrum (GPS) radio sources. Our current understanding of these objects and how they relate to each other and the wider radio-loud AGN population is reviewed in detail by O'Dea (1998) and in other articles in this volume.

A long-standing and open question of the GPS radio sources is, what is the physical mechanism that causes the spectral turnover at $\mathrm{GHz}$ frequencies? Two main competing models are usually invoked to explain the turnover: synchrotron self-absorption (SSA) internal to the source itself, and free-free absorption (FFA) of the radio emission in an external screen of ionised plasma that obscures the source. The aim of this paper is to briefly present an initial investigation and comparison of these two models, as they relate to new radio observations of PKS 1718-649, in particular observations of spectral variability at radio wavelengths above and below the spectral turnover frequency. A more comprehensive discussion of this work will be presented elsewhere (S. J. Tingay et al., in preparation).

PKS 1718-649 (NGC 6328) has been identified as a nearby $(z=0.014$, Fosbury et al., 1977) GPS radio source by Tingay et al. (1997). As a low redshift galaxy, data from previously conducted investigations are readily available in a variety of wavebands. The high quality optical spectra of NGC 6328 (probably the most detailed optical spectral analysis of any GPS or compact steep spectrum (CSS) source) by Filippenko (1985) show that high density $\left(10^{6}-10^{7} \mathrm{~cm}^{-3}\right)$ clouds exist within $\sim 500 \mathrm{pc}$ of the nucleus, and that photoionisation is probably the mechanism responsible for the strong optical emission lines. Keel \& Windhorst (1991) mapped the emission line gas in NGC6328 using narrow-band $\mathrm{H}_{\alpha}+\mathrm{N}[\mathrm{II}]$ observations, revealing a bar-like structure approximately $250 \mathrm{pc} \times 500 \mathrm{pc}$ in extent, oriented north-south and coincident with the radio source.

Australia Telescope Compact Array (ATCA) observations of the neutral hydrogen content of NGC 6328 by Veron-Cetty et al. (1995) revealed a ring structure $37 \mathrm{kpc}$ in diameter with an envelope extending over $180 \mathrm{kpc}$, with kinematics suggestive of a merger involving at least one gas-rich spiral galaxy.

VLBI imaging by Tingay et al. (1997) and more recently Tingay et al. (2002) show the radio structure of PKS 1718-649 to consist of two components, each approximately 2 mas in extent and separated by approximately 7 mas. Tingay et al. (2002) show that the speed of separation of these two components is less than $0.08 c$.

The observational data for PKS 1718-649/NGC 6328 nicely fit the qualitative suggestion that GPS radio sources are confined to parsec scales due to a dense and kinematically complicated AGN environment, possibly due to merger activity. Tingay et al. (1997) applied a quantitative version of this model (Bicknell, Dopita, \& O'Dea 1997, hereafter BDO97) to the PKS 1718-649/NGC 6328 data and found reasonable agreement. In particular, this model suggests that PKS 1718-649 is a young source, less than 100,000 years old, and is confined by a dense environment of $>3 \times 10^{6} \mathrm{~cm}^{-3}$. The model predicts with reasonable success the size and turnover frequency of PKS 1718-649. Such a result, and the optical 
observational data, implies that FFA of the radio emission due to a foreground ionised environment is plausible.

However, even though BD097 derive an analytical expression for GPS source radio spectra, based on FFA by an ensemble of clouds, that reproduces the PKS 1718-649 radio spectrum well, Tingay et al. (1997) point out that since the source size is small for PKS 1718-649, SSA is also a viable explanation of the radio spectrum. The question of the relative contributions made by SSA and FFA to the PKS 1718-649 radio spectrum was left unresolved by Tingay et al. (1997).

Synchrotron self-absorbed sources are optically thick below the turnover, and the flux is set primarily by the source size. Therefore, SSA predicts very little variability below the turnover, even if the relativistic electron distribution, and the flux in the optically thin regime above the turnover, vary in time.

For FFA on the other hand, the spectrum is expected to vary simultaneously and by a similar factor over the entire observed frequency range when the synchrotron source varies intrinsically, and at and below the turnover frequency only when the characteristics of the absorber vary.

Measurements of the radio spectral variability of GPS sources may therefore be able to distinguish between the SSA and FFA mechanisms.

\subsection{Radio Spectral Measurements}

Three observations were made with the ATCA on 2001 July 22, 2002 January 31, and 2002 April 29. These observations used the entire range of frequencies available at the ATCA in all four bands. In each band observations were made at many different frequencies.

At each observing frequency the primary calibrator PKS 1934-638 was observed for approximately 2 min, followed by a 3-5 min observation of PKS 1718-649 at the same frequency. This pattern was repeated over a period of approximately $6 \mathrm{~h}$, until the entire set of observing frequencies had been covered.

Both PKS 1934-638 and PKS 1718-649 are point sources on the ATCA maximum baseline length, which leads to a relatively simple data reduction procedure. Following standard calibration procedures, the data at each frequency were fitted in the $u v$ plane by a point source model to give the flux density of PKS 1718-649 in Stokes $I, Q$, and $U$, and estimates of the errors on these quantities.

In addition, the effects of observing the primary calibrator and target at slightly different elevations have been estimated and accounted for.

Figure 1 shows the results of the observations, the GHz-range spectra of PKS 1718-649 at the three observing epochs. At the higher frequencies, above the spectral turnover, the source flux density increases gradually over the nine month course of the observations. However, below the spectral turnover, the opposite happens: the source gets weaker.

\section{Physical Models of the Spectral Turnover}

In this section we will discuss the two most plausible physical processes that could be responsible for the turnover in the radio spectrum of PKS 1718-649: SSA internal to the radio source, and FFA due to an intervening screen of ionised plasma. These processes have been discussed many times before in the literature, from Kellerman (1966) to more recent reviews of O'Dea (1998) or de Kool \& Begelman (1989).

In the following sections we attempt formal fits of FFA and SSA models to the PKS 1718-649 radio spectra as measured on a flux density scale set by the ATCA primary calibrator, PKS 1934-638.

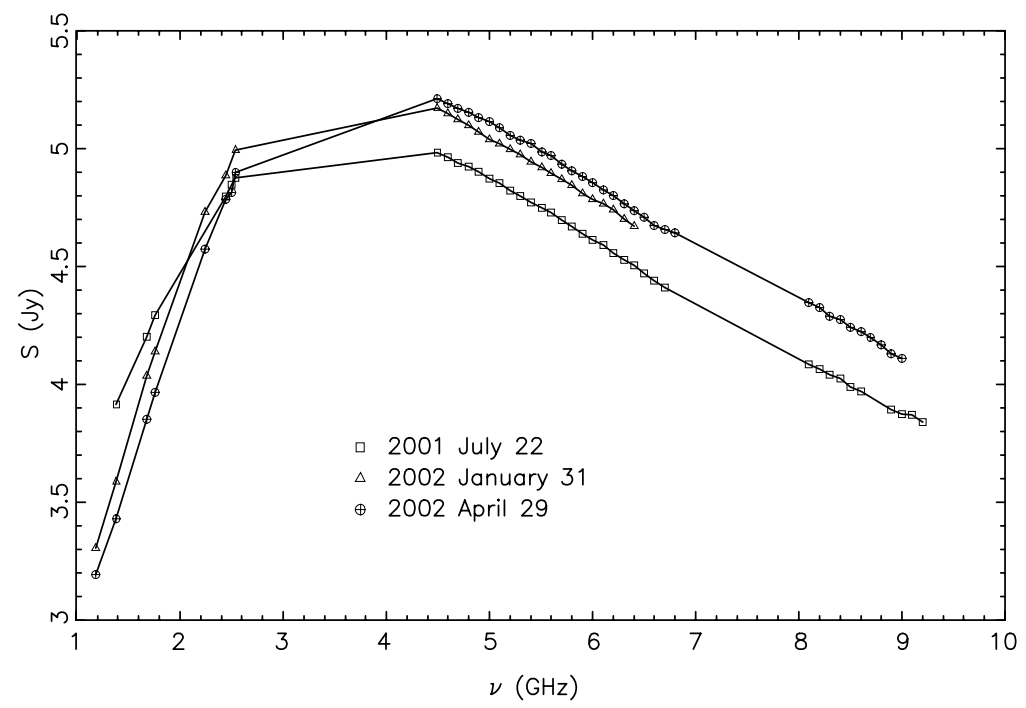

Figure 1 ATCA spectra for PKS 1718-649 obtained at three different epochs, showing the spectral variability. The solid lines shown are not fits to the data but merely connect the points at the different frequencies. 


\subsection{Free-Free Absorption}

Initially we attempted to fit the spectral data from 2001 July 22 with a model consisting of a non-thermal power law spectrum absorbed by a homogeneous free-free absorbing screen. It was immediately obvious that the observed downturn on the low frequency side is much less steep than predicted, and the model fit is totally unacceptable. We have experimented with two ways to resolve this discrepancy.

The first one is to take into account the fact that observations with high spatial resolution (Tingay et al., 1997, 2002) have resolved the source into two components, and the fluxes we have measured in total flux are the sum of these two components. We therefore tried to fit the spectrum with a model consisting of two separate non-thermal power law components, each with its own free-free absorbing screen. It turns out that the spectrum can be well reproduced by two components with very similar luminosities and spectral indices, but with a difference of a factor of 10 in free-free opacity.

The data can be fitted very closely with this sixparameter model, with a formal reduced $\chi^{2}$ of 2.1 . Although the two-component fit does a very good job in the wavelength range considered, it predicts that the spectrum should start to fall very steeply just below $1 \mathrm{GHz}$. This conflicts strongly with other low frequency observations of PKS 1718-649 (Tingay et al., 1997) that indicate a low frequency power law below the turnover frequency.

The presence of a power law at low frequencies is commonly observed in GPS sources, and is not compatible with a homogeneous free-free absorber. However, the FFA model can be rescued if the absorber is allowed to be inhomogeneous, as proposed by BDO97. BDO97 developed a model in which the absorbing screen is inhomogeneous, and parametrise the inhomogeneity by assuming that the screen can be represented by a power law distribution in optical depth. A model spectrum based on these assumptions contains four parameters: the normalisation and slope of the unabsorbed non-thermal spectrum, and the normalisation and slope of the optical depth distribution. We fitted such a model to the new observations presented here. This model is very successful in reproducing the observations. Not only does it provide a better fit to the 2001 July 22 observations (a reduced $\chi^{2}$ of 1.1) with fewer free parameters, but it also predicts the low frequency power law very well.

Given that this inhomogeneous free-free absorber model is so successful in reproducing the 2001 July 22 data, we also examined the fit of the model to the other two epochs of spectral data. In both the 2002 January 31 and 2002 April 29 datasets, the fit of the model to the data were very good (with different sets of free parameters). A much more detailed description of this analysis, including a graphical presentation of the fits to the data, is to be presented in a more comprehensive paper (S. J. Tingay et al., in preparation). We present the initial, basic results of our analyses here. Table 1 gives the parameters of the BDO97 model fit to the data at the three epochs.

If this model is correct, a comparison of the resulting model parameters over the three epochs indicates that the intrinsic power of the synchrotron emission from the source has increased over the course of the three epochs, while the intrinsic spectrum has slightly flattened. This is most easily seen in the spectra shown in Figure 1 above the turnover, at $5 \mathrm{GHz}$ and above. The flux density at these frequencies is clearly rising. A rise in the intrinsic power of the source with a flattening of the spectrum could be consistent with the enhanced emission expected from reaccelerated electrons in a shock in the flow of jet material.

However, at lower frequencies, a change in the optical depth distribution of the free-free absorbing clouds is required to explain the decrease in the flux density below the turnover frequency.

We can speculate as to what form this evolution could take. The clouds could be in motion in a direction perpendicular to our line of sight to the source, causing clouds to be added to and removed from the ensemble that obscure the source. Similarly, the free-free clouds could be quasistationary and the radio source itself could be expanding, so that different parts of the free-free screen are obscuring the radio source as the radio source expands. Estimates of likely cloud speeds near the nucleus (Filippenko 1985) and limits on the speed of separation of the milliarcsecondscale radio components (Tingay et al., 2002) would appear to rule these suggestions out.

Table 1. Best-fit parameters of the BDO97 model for the PKS 1718-649 radio spectral data collected from the ATCA*

\begin{tabular}{lcccccc}
\hline Date & $A$ & $p$ & $\nu_{0}$ & $\alpha$ & Reduced $\chi^{2}$ & d.o.f. \\
\hline 2001 July 22 & 6.70 & -0.53 & 4.05 & 0.60 & 1.1 & 34 \\
2002 Jan 31 & 7.27 & -0.43 & 3.53 & 0.57 & 0.9 & 21 \\
2002 Apr 29 & 7.30 & -0.41 & 3.72 & 0.58 & 2.5 & 36 \\
\hline
\end{tabular}

* The parameters are: $A$ - the amplitude of the intrinsic synchrotron spectrum; $p-$ the slope of the power law distribution of free-free absorbing clouds; $v_{0}$ - characteristic of the maximum free-free optical depth in the distribution of optical depths; $\alpha$ - the spectral index of the intrinsic synchrotron spectrum. Full descriptions of the parameters and the derivation of the model appear in BDO97. 
Alternatively, the free-free absorbing clouds could be considered quasi-stationary, but evolving internally. This may be possible if the clouds are interacting with the jet that presumably powers the radio source. A small number of high density clouds may be disrupted, leaving a greater number of clouds with smaller free-free optical depths. This scenario is obviously difficult to quantify but may be consistent with the increase in the high frequency flux density and the flattening of the intrinsic spectrum, also indicated by the model. The shock that could cause the high frequency changes may be due to a mechanical interaction between the jet and the external free-free absorbing medium, causing a modification of the properties of the free-free absorbing clouds.

\subsection{Synchrotron Self-Absorption}

The other main contender for the physical mechanism responsible for the spectral turnovers in GPS sources is SSA (e.g. Kellerman 1966).

The peak in the spectrum of a single homogeneous SSA source with a power law relativistic electron distribution is much narrower than observed in PKS 1718-649, just as in the case of FFA. We can again try to overcome this problem by fitting the spectra with two SSA components. The fit is worse than the corresponding FFA fits, and also suffers from the problem of falling too steeply below $1 \mathrm{GHz}$. This does not allow us to reject SSA as a viable explanation, since it is well known that inhomogeneity and radiation transfer effects in the source always tend to flatten the spectrum below the turnover.

There is an argument that strongly favors SSA for PKS 1718-649. For SSA sources there is a theoretical relation between the peak frequency, size, magnetic field, and peak flux density of the source (e.g. Kellerman \& Pauliny-Toth 1981). For PKS 1718-649 we know both the peak frequency and flux density from the spectra, and the size from the observations of Tingay et al. (2002). Using the theoretical relation, this allows us to estimate the magnetic field in the source at $10^{-2} \mathrm{G}$ if the turnover is due to SSA. This is a very reasonable value for the magnetic field at a distance of $\sim 1 \mathrm{pc}$ from the active nucleus.

However, variability of the radio spectrum at frequencies below the turnover frequency posses a difficulty for SSA models. For SSA, the source is optically thick below the turnover, and the flux is set by the source size. Therefore, SSA predicts very little variability below the turnover, even if the relativistic electron distribution, and the flux in the optically thin regime above the turnover, vary in time.

An increasing flux below the spectral peak can be explained by an expanding radio source. However,
Figure 1 shows that the flux below the peak is decreasing rather than increasing, implying that the source is contracting rather than expanding. Other observations of GPS sources show the radio sources to be separating in general (e.g. the results presented by Polatidis \& Conway 2003), implying that the individual components in the sources are also expanding. If the GPS and CSS sources evolve into large-scale FR I or FR II sources, then expansion must dominate contraction over their lifetimes. Of course, the VLBI data do not allow us to rule out in this individual case that the components in PKS 1718-649 are contracting. Models based on SSA may have to be much more complicated than those presented here to be able to reproduce the data shown in Figure 1.

\section{Conclusions}

From the discussion above it appears clear that the shape of the spectral turnover, combined with possible inhomogeneity in the source and absorber, and the observed spectral variability still do not allow us to put hard constraints on the underlying physical mechanism causing the GPS in PKS 1718-649. In either case, however, it would seem that novel models will have to be produced in order to explain the body of data. SSA appears to be a plausible candidate simply because an SSA turnover is expected where the peak is observed, based on the peak flux, source size and a reasonable magnetic field. However, the spectral variability would appear to be naturally explained by the FFA that is expected based on optical observations of the nucleus of the galaxy.

More work is clearly needed on this interesting source. A detailed discussion of the initial results and ideas presented here will appear elsewhere (S. J. Tingay et al., in preparation).

\section{References}

Bicknell, G. V., Dopita, M. A., \& O’Dea, C. P. 1997, APJ, 485, 112 (BDO97)

de Kool, M., \& Begelman, M. C. 1989, APJ, 345, 135

Filippenko, A. V. 1985, APJ, 289, 475

Fosbury, R. A. E., Mebold, U., Goss, W. M., \& van Woerden, H. 1977, MNRAS, 179, 89

Keel, W. C., \& Windhorst, R. A. 1991, APJ, 383, 135

Kellerman, K. I. 1966, AuJPh, 19, 195

Kellerman, K. I., \& Pauliny-Toth, I. I. K. 1981, ARA\&A, 19,373

O’Dea, C. P. 1998, PASP, 110, 493

Polatidis, A. G., \& Conway, J. E. 2003, PASA, 20, 69

Tingay, S. J., et al. 1997, AJ, 113, 2025

Tingay, S. J., et al. 2002, APJS, 141, 311

Véron-Cetty, M. P., Woltjer, L., Ekers, R. D., \& Staveley-Smith, L. 1995, A\&A, 297, L79 